\title{
INFINITE DIMENSIONAL FUNCTIONAL CONVERGENCES IN RANDOM BALLS MODEL *
}

\author{
JeAn-Christophe Breton ${ }^{1}$ And Renan Gobard ${ }^{1}$
}

\begin{abstract}
We consider a weighted random ball model generated by a Poisson measure. The macroscopic behaviour of the weight amassed on this model by a configuration has recently received attention. In this paper, we complement the previous finite dimensional distribution fluctuation results and propose functional convergences of such functionals on the set of configurations.
\end{abstract}

Mathematics Subject Classification. 60F17, 60G60, 60G18, 60H05.

Received December 21, 2014. Revised July 10, 2015.

\section{INTRODUCTION}

We consider a generalized random balls model in $\mathbb{R}^{d}$ generated by a Poisson point process $N_{\lambda}$ on $\mathbb{R}^{d} \times \mathbb{R}_{+} \times \mathbb{R}$ with intensity

$$
n_{\lambda}(\mathrm{d} \mathbf{x}, \mathrm{d} r, \mathrm{~d} m)=\lambda \mathrm{d} \mathbf{x} F(\mathrm{~d} r) G(\mathrm{~d} m)
$$

where $\lambda \in \mathbb{R}_{+}$and $F, G$ are probability measures respectively on $\mathbb{R}_{+}$and $\mathbb{R}$. The most natural way to deal with the triplets $(\mathbf{x}, r, m)$ is to see them as weighted random balls with center $\mathbf{x}$, radius $r$ and weight $m$. The parameter $\lambda$ stands for the intensity of the number of ball since the mean number of balls in, say, a measurable bounded set $A \subset \mathbb{R}^{d}$ with Lebesgue measure 1 is $\mathbb{E}\left[N_{\lambda}\left(A \times \mathbb{R}^{+} \times \mathbb{R}\right)\right]=\lambda$. Random balls models of this type are typically used to represent spatial communication networks where the centers $\mathbf{x}$ are antennas transmitting a signal with a range of emission $r$. The weight $m$ then represents the intensity of the signal (see $[6,13])$. From a modeling standpoint, it is natural to consider that the signal fades out when it goes away from its emitting station $\mathbf{x}$. Representing such a fading effect by a shape function $h$, the signal of intensity $m$ emitted in $\mathbf{x}$ is received in some $\mathbf{y}$ with intensity $m h\left(r^{-1}(\mathbf{x}-\mathbf{y})\right)$, in this picture the quantity $r$ plays now the role of the rate of the fading. If we assume that no interference occurs between the different stations, the quantity of signal received in some $\mathbf{y} \in \mathbb{R}^{d}$ is then given by:

$$
M_{\lambda}(\mathbf{y})=\int_{\mathbb{R}^{d} \times \mathbb{R}_{+} \times \mathbb{R}} m h\left(r^{-1}(\mathbf{y}-\mathbf{x})\right) N_{\lambda}(\mathrm{d} \mathbf{x}, \mathrm{d} r, \mathrm{~d} m) .
$$

Keywords and phrases. Self-similarity, generalized random fields, functional convergence, tightness, Poisson point process.

* Research partially supported by the Centre Henri Lebesgue ("Investissements d'avenir" program - ANR-11-LABX-0020-01).

1 Institut de recherche mathématique de Rennes (IRMAR), UMR 6625, Université de Rennes 1, Campus de Beaulieu, 263 Avenue du Général Leclerc, 35042 Rennes, France. jean-christophe.breton@univ-rennes1.fr; renan.gobard@univ-rennes1.fr 
Extending the quantity defined in (1.2) to a configuration represented by a measure $\mu$, we consider now

$$
M_{\lambda}(\mu)=\int_{\mathbb{R}^{d}} M_{\lambda}(\mathbf{y}) \mu(\mathrm{d} \mathbf{y})=\int_{\mathbb{R}^{d} \times \mathbb{R}_{+} \times \mathbb{R}} m \mu\left[\tau_{\mathbf{x}, r} h\right] N_{\lambda}(\mathrm{d} \mathbf{x}, \mathrm{d} r, \mathrm{~d} m)
$$

where we set $\tau_{\mathbf{x}, r} h(\mathbf{y})=h\left(r^{-1}(\mathbf{x}-\mathbf{y})\right)$ and $\mu[f]=\int_{\mathbb{R}^{d}} f(\mathbf{y}) \mu(\mathrm{d} \mathbf{y})$, see [3]. Since there is no restriction to consider only positive measures, we consider configurations $\mu$ in the set $\mathbb{Z}\left(\mathbb{R}^{d}\right)$ of signed measures on $\mathbb{R}^{d}$ with finite total variation. Recall that the total variation $\|\mu\|_{T V}=\sup _{A \in \mathcal{B}\left(\mathbb{R}^{d}\right)}|\mu(A)|$ defines a norm on $\mathcal{Z}\left(\mathbb{R}^{d}\right)$ and that equipped with this norm, $\mathcal{Z}\left(\mathbb{R}^{d}\right)$ is a Banach space. This model is the one studied in [3]. When $h=\mathbf{1}_{B(\mathbf{0}, 1)}$, we recover the setting of $[1,2,9]$.

We specify now the hypotheses on $G, F$ and $h$. The measure $G$ drives the behaviour of the weights, it is assumed to be in the domain of attraction of an $\alpha$-stable law with $\alpha \in(1,2]$, see [10]. According to ([5], Sect. XVII.5), this is equivalent to the following estimate on the characteristic function $\varphi_{G}$ of $G$ :

$$
\varphi_{G}(\theta)=1+i \theta \tau-\sigma^{\alpha}|\theta|^{\alpha}(1+i b \varepsilon(\theta) \tan (\pi \alpha / 2))+o\left(|\theta|^{\alpha}\right), \quad \text { as } \theta \rightarrow 0,
$$

where $\varepsilon(\theta)=1$ if $\theta>0, \varepsilon(\theta)=-1$ if $\theta<0$ and $\varepsilon(0)=0$. In the case $\alpha \in(1,2)$, a typical choice for $G$ is a heavy-tailed distribution while for $\alpha=2, G$ may be any distribution with finite variance. Observe that since we consider $\alpha>1$, the measure $G$ has a finite moment of order 1 . The probability measure $F$ drives the behaviour of the radius $r$ (actually the fading rate in the model described above); we assume $F$ has a regularly varying tail with index $\beta \in(d, \alpha d)$, that is:

$$
\bar{F}(r)=\int_{r}^{+\infty} F(\mathrm{~d} u) \sim_{r \rightarrow+\infty} C_{\beta} r^{-\beta} .
$$

The condition (1.5) ensures that the mean volume of the random balls is finite and so is the expectation of $M_{\lambda}(\mu)$ for every $\mu$ with a finite total variation, see [3]. On the contrary, $\beta<2 d$ implies that the variance of the law of the volume is infinite.

Since in our interpretation, the shape function $h$ represents a fading effect, it is natural to consider that $h$ is radial and (radially) decreasing. The function $h$ thus generalizes the range of the emission. As discussed above, the choice $h=\mathbf{1}_{B(\mathbf{0}, 1)}$ recovers the classical setting of random ball $B(\mathbf{x}, r)$ with weight $m$ as investigated in $[1,2,9]$. However, from a mathematical standpoint, it is not required to assume that $h$ is decreasing or radial and more complex interfering phenomena can thus be investigated.

In what follows, we are interested in the macroscopic analysis of such model. It consists in investigating the behaviour of $M_{\lambda}(\mu)$ when a zooming-out is performed, i.e. apply the change $r \mapsto \rho r$ in the model and consider the resulting changed field

$$
M_{\lambda, \rho}(\mu)=\int_{\mathbb{R}^{d} \times \mathbb{R}_{+} \times \mathbb{R}} m \mu\left[\tau_{\mathbf{x}, r} h\right] N_{\lambda, \rho}(\mathrm{d} \mathbf{x}, \mathrm{d} r, \mathrm{~d} m),
$$

where $N_{\lambda, \rho}$ is the Poisson random measure with intensity $n_{\lambda, \rho}=\lambda \mathrm{d} x F_{\rho}(\mathrm{d} r) G(\mathrm{~d} m)$ and $F_{\rho}$ is the image measure of $F$ by $r \mapsto \rho r$. When the intensity $\lambda(\rho)$ is adapted accordingly to the rate $\rho$ of zooming-out, [3] describes two limiting behaviours obtained when a centering and a suitable normalization $n(\rho)$ are applied to $M_{\lambda(\rho), \rho}(\mu)$. Actually, due to the linear structure of $M_{\lambda(\rho), \rho}(\mu)$ in $\mu$, the limits are obtained in the finite dimensional distributions ( $f d d)$ sense (see Sect. 2 for precise conditions). For the two scaling regimes, functional convergences are also obtained in [3] but only for a finite dimensional subfamily of configurations $\mu$. The goal of the present note is to complete the results of [3] for configurations in an infinite dimensional family $\mathcal{A} \subset \mathcal{Z}\left(\mathbb{R}^{d}\right)$ and to provide functional convergences in the space $\mathcal{C}(\mathcal{A})$ of continuous functions in $\mathcal{A}$. Since $f d d$ convergences are already proved in [3] for smooth configurations, the present note really focuses on tightness and to that purpose we will assume that

$$
\mathcal{A} \text { is a compact subset in } L^{1}\left(\mathbb{R}^{d}\right) \cap L^{\alpha}\left(\mathbb{R}^{d}\right) \text { for the }\|\cdot\|_{T V} \text { topology, }
$$


where we shall (abusively) note $L^{1}\left(\mathbb{R}^{d}\right) \cap L^{\alpha}\left(\mathbb{R}^{d}\right)$ the space of smooth measures $\mu(\mathrm{d} \mathbf{y})=\phi_{\mu}(\mathbf{y})$ dy such that $\phi_{\mu} \in L^{1}\left(\mathbb{R}^{d}\right) \cap L^{\alpha}\left(\mathbb{R}^{d}\right)$. Remark that for configurations in $L^{1}\left(\mathbb{R}^{d}\right)$, the $L^{1}$ norm corresponds to the total variation norm. The note is organized as follows: Section 2 contains our main conditions and results. The proof is given in Section 3 and discussions on our hypotheses are provided in Section 4 with examples. Technical results are postponed in Appendix A.

\section{MAIn RESUlTS}

First, we recall the $f d d$ results obtained in [3]. The shape function $h$ is assumed to be continuous almost everywhere and such that

$$
h^{*}(\mathbf{x}):=\sup \{|h(r \mathbf{x})| r \geq 1\} \in L^{1}\left(\mathbb{R}^{d}\right) \cap L^{\alpha}\left(\mathbb{R}^{d}\right) .
$$

Note that this implies $h \in L^{1}\left(\mathbb{R}^{d}\right) \cap L^{\alpha}\left(\mathbb{R}^{d}\right)$ and note that if $h$ is radially non-increasing, then $h^{*}=h$. Throughout, we note $\stackrel{f d d}{\Longrightarrow}$ the convergence in finite-dimensional law in $L^{1}\left(\mathbb{R}^{d}\right) \cap L^{\alpha}\left(\mathbb{R}^{d}\right)$. Proposition 2.1 in [3] gives the limiting behaviour, when $\rho \rightarrow 0$, of

$$
\widetilde{M}_{\rho}(\mu)=n(\rho)^{-1}\left(M_{\lambda(\rho), \rho}(\mu)-\mathbb{E}\left[M_{\lambda(\rho), \rho}(\mu)\right]\right) .
$$

(1) (Large regime) If $\lambda(\rho) \rho^{\beta} \rightarrow+\infty$, then, setting $n(\rho)=\left(\lambda(\rho) \rho^{\beta}\right)^{1 / \alpha}$, we have:

$$
\widetilde{M}_{\rho}(\cdot) \stackrel{f d d}{\Longrightarrow} Z_{\alpha}(\cdot)
$$

when $\rho \rightarrow 0$ and where $Z_{\alpha}$ is the stable field

$$
Z_{\alpha}(\mu)=\int_{\mathbb{R}^{d} \times \mathbb{R}_{+}} \mu\left[\tau_{\mathbf{x}, r} h\right] M_{\alpha}(\mathrm{d} \mathbf{x}, \mathrm{d} r)
$$

with respect to the $\alpha$-stable measure $M_{\alpha}$ with control measure $\sigma^{\alpha} C_{\beta} r^{-1-\beta} \mathrm{d} \mathbf{x} \mathrm{d} r$ and constant skewness function $b$, where $\sigma$ and $b$ are related to $G$ by (1.4).

(2) (Intermediate regime) If $\lambda(\rho) \rho^{\beta} \rightarrow a$ for some $a \in(0,+\infty)$, then, setting $n(\rho)=1$, we have:

$$
\widetilde{M}_{\rho}(\cdot) \stackrel{f d d}{\Longrightarrow} J_{a}(\cdot)
$$

when $\rho \rightarrow 0$ and where $J_{a}$ is the compensated Poisson integral

$$
J_{a}(\mu)=\int_{\mathbb{R}^{d} \times \mathbb{R} \times \mathbb{R}_{+}} m \mu\left[\tau_{\mathbf{x}, r} h\right] \widetilde{N}_{\beta, a}(\mathrm{~d} \mathbf{x}, \mathrm{d} r, \mathrm{~d} m)
$$

with respect to the compensated Poisson random measure $\widetilde{N}_{\beta, a}$ with intensity $a C_{\beta} r^{-\beta-1} \mathrm{~d} \mathbf{x} \mathrm{d} r G(\mathrm{~d} m)$.

In order to reinforce $(2.1)$ and $(2.2)$ into functional convergences in $\mathcal{C}(\mathcal{A})$, we shall assume the following uniform control of the measures $\mu \in \mathcal{A}$ by some measure $\mu^{*}(\mathbf{x}) \mathrm{d} \mathbf{x}$ :

$$
\sup _{\mu \in \mathcal{A}}\left(\int_{\mathbb{R}^{d}}\left|h\left(r^{-1}(\mathbf{y}-\mathbf{x})\right)\right|^{p}|\mu|(\mathrm{d} \mathbf{y})\right) \leq \int_{\mathbb{R}^{d}}\left|h\left(r^{-1}(\mathbf{y}-\mathbf{x})\right)\right|^{p} \phi^{*}(\mathbf{y})(\mathrm{d} \mathbf{y})
$$

for all $\mathbf{x} \in \mathbb{R}^{d}$ and all $r>0$, with $\phi^{*} \in L^{1}\left(\mathbb{R}^{d}\right) \cap L^{\alpha}\left(\mathbb{R}^{d}\right)$ and $p \geq 1$. A discussion on $\left(C_{p}\right)$ is given in Section 4 . More precisely, we assume below that $\left(C_{p}\right)$ holds true for $p=1$ and for $p$ equal to some (arbitrary) $\gamma \in(1, \alpha)$. 
Our main result states as follows:

Proposition 2.1. Assume that the family $\mathcal{A}$ of measures satisfies $(C 0)$ and $\left(C_{p}\right)$ for $p \in\{1, \gamma\}$ for some $1<\gamma<\alpha$ and that the shape function $h$ satisfies $(H)$.

(1) (Large regime) If $\lambda(\rho) \rho^{\beta} \rightarrow+\infty$, then:

$$
\widetilde{M}_{\rho}(\cdot) \stackrel{\mathcal{C}(\mathcal{A})}{\Longrightarrow} Z_{\alpha}(\cdot), \quad \rho \rightarrow 0 .
$$

(2) (Intermediate regime) If $\lambda(\rho) \rho^{\beta} \rightarrow a$ for some $a \in(0,+\infty)$, then:

$$
\widetilde{M}_{\rho}(\cdot) \stackrel{\mathcal{C}(\mathcal{A})}{\Longrightarrow} J_{a}(\cdot), \quad \rho \rightarrow 0 .
$$

Another interesting finite-dimensional result is Proposition 2.13 in [2] which states that when $a \rightarrow+\infty$, we have

$$
a^{-1 / \alpha} J_{a}(\cdot) \stackrel{f d d}{\Longrightarrow} Z_{\alpha}(\cdot) .
$$

We can again reinforce (2.5) into functional convergence:

Proposition 2.2. Under the same hypotheses as in Proposition 2.1, we have:

$$
a^{-1 / \alpha} J_{a}(\cdot) \stackrel{\mathcal{C}(\mathcal{A})}{\Longrightarrow} Z_{\alpha}(\cdot), \quad a \rightarrow+\infty .
$$

Remark 2.3. When the shape function $h$ is bounded (which is typically the case when $h$ is radially nonincreasing), we can assume condition $\left(C_{p}\right)$ only for $p=1$ in Propositions 2.1 and 2.2 (no $\left(C_{\gamma}\right.$ ) for some $\gamma \in(1, \alpha)$ is required). To see this, observe that in the proof below (Sect. 3), $\left(C_{\gamma}\right)$ is used only in the proof of (1) in Lemma A.1 which can be replaced by (2) in Lemma A.1 when $h$ is bounded.

As a consequence of our results, for all continuous functional $f$ on $\mathcal{C}(\mathcal{A})$, we have

$$
f\left(\widetilde{M}_{\rho}(\cdot)\right) \Longrightarrow f\left(Z_{\alpha}(\cdot)\right), \quad \rho \rightarrow 0
$$

or

$$
f\left(\widetilde{M}_{\rho}(\cdot)\right) \Longrightarrow f\left(J_{a}(\cdot)\right), \quad \rho \rightarrow 0
$$

depending on the regime considered. For instance, with $f(x)=\sup _{\mu \in \mathcal{A}}|x(\mu)|$ we can use (2.7) and (2.8) to detect excessive fluctuations in the model.

\section{Proofs}

Proof of Proposition 2.1. It is well known that the functional convergence is equivalent to the combination of the $f d d$ convergences and tightness. Since the $f d d$ convergences is known $(c f .(2.1)$ and (2.2)), it remains to prove the tightness of $\left(M_{\rho}(\mu)\right)_{\mu \in \mathcal{A}}$. Thanks to Prohorov's theorem, this is equivalent to relative compactness and this will be obtained, using Arzelà-Ascoli's theorem for $\mathcal{C}(\mathcal{A})$, by showing the following control of the uniform modulus of continuity (see [8], Thm. A2.1):

$$
\forall \varepsilon>0, \lim _{\delta \rightarrow 0} \sup _{\rho>0} \mathbb{P}\left(\sup _{\substack{\left\|\mu_{1}-\mu_{2}\right\|_{T V} \leq \delta \\ \mu_{1}, \mu_{2} \in \mathcal{A}}}\left|\widetilde{M}_{\rho}\left(\mu_{1}\right)-\widetilde{M}_{\rho}\left(\mu_{2}\right)\right|>\varepsilon\right)=0 .
$$

We derive (3.1) with Markov's inequality by proving that for $1<\gamma<\alpha$ given in Proposition 2.1:

$$
\lim _{\delta \rightarrow 0} \sup _{\rho>0} \mathbb{E}\left[\sup _{\substack{\left\|\mu_{1}-\mu_{2}\right\|_{T V} \leq \delta \\ \mu_{1}, \mu_{2} \in \mathcal{A}}}\left|\widetilde{M}_{\rho}\left(\mu_{1}\right)-\widetilde{M}_{\rho}\left(\mu_{2}\right)\right|^{\gamma}\right]=0 .
$$


Our argument to prove (3.2) exploits moment bounds obtained in [3] for such random field $\left(M_{\lambda, \rho}(\mu)\right)_{\mu \in \mathcal{A}}$. We use in particular the following expression of a fractional moment from [12]: if $X$ is a random variable with characteristic function $\varphi_{X}(t)=\mathbb{E}[\exp (i t X)]$, then for $1<\gamma<2$ :

$$
\mathbb{E}\left[|X|^{\gamma}\right]=A(\gamma) \int_{0}^{+\infty}\left(1-\left|\varphi_{X}(\theta)\right|^{2}\right) \theta^{-1-\gamma} \mathrm{d} \theta
$$

where

$$
A(\gamma)=\left(\int_{0}^{+\infty}(1-\cos (x)) x^{-1-\gamma} \mathrm{d} \mathbf{x}\right)^{-1}<+\infty .
$$

In order to be in position to exploit (3.3) for the fractional moment in (3.2) we need to specify the corresponding characteristic function. To that purpose, we first bound $\sup _{\substack{\left\|\mu_{1}-\mu_{2}\right\|_{T V} \leq \delta \\ \mu_{1}, \mu_{2} \in \mathcal{A}}}\left|\widetilde{M}_{\rho}\left(\mu_{1}\right)-\widetilde{M}_{\rho}\left(\mu_{2}\right)\right|$ as follows:

$$
\begin{aligned}
\sup _{\substack{\left\|\mu_{1}-\mu_{2}\right\|_{T V} \leq \delta \\
\mu_{1}, \mu_{2} \in \mathcal{A}}}\left|\widetilde{M}_{\rho}\left(\mu_{1}\right)-\widetilde{M}_{\rho}\left(\mu_{2}\right)\right| \leq & \sup _{\substack{\left\|\mu_{1}-\mu_{2}\right\|_{T V} \leq \delta \\
\mu_{1}, \mu_{2} \in \mathcal{A}}}\left|M_{\lambda, \rho}\left(n(\rho)^{-1}\left(\mu_{1}-\mu_{2}\right)\right)\right| \\
& +\mathbb{E}\left[\sup _{\substack{\left\|\mu_{1}-\mu_{2}\right\|_{T V} \leq \delta \\
\mu_{1}, \mu_{2} \in \mathcal{A}}}\left|M_{\lambda, \rho}\left(n(\rho)^{-1}\left(\mu_{1}-\mu_{2}\right)\right)\right|\right] .
\end{aligned}
$$

Since for $\gamma>1$

$$
\mathbb{E}\left[(|X|+\mathbb{E}[|X|])^{\gamma}\right] \leq 2^{\gamma} \mathbb{E}\left[|X|^{\gamma}\right],
$$

we shall establish (3.2) by applying (3.3) to

$$
X=\sup _{\substack{\left\|\mu_{1}-\mu_{2}\right\|_{T V} \leq \delta \\ \mu_{1}, \mu_{2} \in \mathcal{A}}} \mid M_{\lambda, \rho}\left(n(\rho)^{-1}\left(\mu_{1}-\mu_{2}\right) \mid\right.
$$

We have

$$
\sup _{\substack{\left\|\mu_{1}-\mu_{2}\right\|_{T V} \leq \delta \\ \mu_{1}, \mu_{2} \in \mathcal{A}}}\left|M_{\lambda, \rho}\left(n(\rho)^{-1}\left(\mu_{1}-\mu_{2}\right)\right)\right| \leq \widehat{M}_{\rho}\left(n(\rho)^{-1} \mathfrak{m}_{\delta}\right):=\int|m| n(\rho)^{-1} \mathfrak{m}_{\delta}\left[\tau_{\mathbf{x}, r}|h|\right] N_{\lambda(\rho), \rho}(\mathrm{d} \mathbf{x}, \mathrm{d} r, \mathrm{~d} m)
$$

where

$$
\mathfrak{m}_{\delta}\left[\tau_{\mathbf{x}, r}|h|\right]=\sup _{\substack{\left\|\mu_{1}-\mu_{2}\right\|_{T V} \leq \delta \\ \mu_{1}, \mu_{2} \in \mathcal{A}}}\left|\mu_{1}-\mu_{2}\right|\left[\tau_{\mathbf{x}, r}|h|\right]
$$

(observe that $\mathfrak{m}_{\delta}$ is not a measure a priori). Since $\widehat{M}_{\rho}\left(n(\rho)^{-1} \mathfrak{m}_{\delta}\right)$ is a (non centered) Poisson integral, its characteristic function is given by:

$$
\varphi_{\widehat{M}_{\rho}\left(n(\rho)^{-1} \mathfrak{m}_{\delta}\right)}(\theta)=\exp \left(\int_{\mathbb{R}^{d} \times \mathbb{R}_{+}} \psi_{G}\left(n(\rho)^{-1} \theta \mathfrak{m}_{\delta}\left[\tau_{\mathbf{x}, r}|h|\right]\right) \lambda(\rho) \mathrm{d} \mathbf{x} F_{\rho}(\mathrm{d} r)\right)
$$


with $\psi_{G}(u)=\int_{\mathbb{R}}\left(\mathrm{e}^{i u|m|}-1\right) G(\mathrm{~d} m)$. Note also $\Psi_{G}(u)=\int_{\mathbb{R}}\left(\mathrm{e}^{i u|m|}-1-i u|m|\right) G(\mathrm{~d} m)$ and observe that $\operatorname{Re}\left(\Psi_{G}\right)=$ $\operatorname{Re}\left(\psi_{G}\right)$ (to be used below in (3.8)). Hence,

$$
\begin{aligned}
1-\left|\varphi_{\widehat{M}_{\rho}\left(n(\rho)^{-1} \mathfrak{m}_{\delta}\right)}(\theta)\right|^{2} & =1-\exp \left(\int_{\mathbb{R}^{d} \times \mathbb{R}_{+}} 2 \operatorname{Re}\left(\Psi_{G}\left(n(\rho)^{-1} \theta \mathfrak{m}_{\delta}\left[\tau_{\mathbf{x}, r}|h|\right]\right)\right) \lambda(\rho) \mathrm{d} \mathbf{x} F_{\rho}(\mathrm{d} r)\right) \\
& \leq 1-\exp \left(-2 \int_{\mathbb{R}^{d} \times \mathbb{R}_{+}}\left|\Psi_{G}\left(n(\rho)^{-1} \theta \mathfrak{m}_{\delta}\left[\tau_{\mathbf{x}, r}|h|\right]\right)\right| \lambda(\rho) \mathrm{d} \mathbf{x} F_{\rho}(\mathrm{d} r)\right) \\
& \leq 1-\exp \left(-2 C(G) n(\rho)^{-\alpha} \lambda(\rho)|\theta|^{\alpha} \int_{\mathbb{R}^{d} \times \mathbb{R}_{+}}\left|\mathfrak{m}_{\delta}\left[\tau_{\mathbf{x}, r}|h|\right]\right|^{\alpha} \mathrm{d} \mathbf{x} F_{\rho}(\mathrm{d} r)\right)
\end{aligned}
$$

using the local behaviour (1.4) of $\Psi_{G}$ in 0. Using Lemma A.3 in the Appendix with $\gamma:=\alpha>\beta / d$ therein, we have

$$
\int_{\mathbb{R}^{d} \times \mathbb{R}_{+}}\left|\mu\left[\tau_{\mathbf{x}, r}|h|\right]\right|^{\alpha} \mathrm{d} \mathbf{x} F_{\rho}(\mathrm{d} r) \leq C\left(\phi^{*}\right) \rho^{\beta} \delta^{\frac{\alpha d-\beta}{d}}
$$

where

$$
C\left(\phi^{*}\right)=2^{\frac{\alpha d-\beta}{(\alpha-1) d}} \frac{(\alpha-1) d}{(\beta-d)(\alpha d-\beta)}\left(\left\|\phi^{*}\right\|_{\alpha}\|h\|_{1}\right)^{\frac{\alpha(\beta-d)}{(\alpha-1) d}}\left(\left\|\phi^{*}\right\|_{1}^{1 / \alpha}\|h\|_{\alpha}\right)^{\frac{(\alpha d-\beta) \alpha}{(\alpha-1) d}} .
$$

Plugging the bounds (3.9) and (3.10) into (3.3) with $X$ as in (3.6) and combining with (3.4) and (3.5), we obtain

$$
\begin{aligned}
\mathbb{E}\left[\left|\sup _{\substack{\left\|\mu_{1}-\mu_{2}\right\|_{T V} \leq \delta \\
\mu_{1}, \mu_{2} \in \mathcal{A}}}\right| \widetilde{M}_{\rho}\left(\mu_{1}-\widetilde{M}_{\rho}\left(\mu_{2}\right)||^{\gamma}\right]\right. & \leq A(\gamma) \int_{0}^{+\infty}\left(1-\exp \left(-2 C(G) n(\rho)^{-\alpha} \lambda(\rho) \rho^{\beta}|\theta|^{\alpha} C\left(\phi^{*}\right) \delta^{\frac{\alpha d-\beta}{d}}\right)\right) \theta^{-1-\gamma} \mathrm{d} \theta \\
& =A(\gamma) A(\alpha, \gamma)\left(n(\rho)^{-\alpha} \lambda(\rho) \rho^{\beta}\right)^{\gamma / \alpha}\left(2 C(G) C\left(\phi^{*}\right)\right)^{\gamma / \alpha} \delta^{\frac{(\alpha d-\beta) \gamma}{\alpha d}}
\end{aligned}
$$

with a straightforward change of variables to derive (3.11) and where

$$
A(\alpha, \gamma)=\int_{0}^{+\infty}\left(1-\exp \left(-\theta^{\alpha}\right)\right) \theta^{-1-\gamma} \mathrm{d} \theta
$$

which is finite for $\gamma<\alpha$.

In the large regime $n(\rho)=\left(\lambda(\rho) \rho^{\beta}\right)^{1 / \alpha}$ and the bound (3.11) entails (3.2) while in the intermediate regime $n(\rho)^{-\alpha} \lambda(\rho) \rho^{\beta}=\lambda(\rho) \rho^{\beta}$ is bounded so that (3.11) still entails (3.2). In both cases, this concludes the Proof of Proposition 2.1.

Proof of Proposition 2.2. In order to prove Proposition 2.2, we follow the same lines of reasoning as in the previous proof. Set

$$
I_{a}(\mu)=\int_{\mathbb{R}^{d} \times \mathbb{R} \times \mathbb{R}_{+}} a^{-1 / \alpha} m \mu\left[\tau_{\mathbf{x}, r} h\right] N_{\beta, a}(\mathrm{~d} \mathbf{x}, \mathrm{d} r, \mathrm{~d} m),
$$

where $N_{\beta, a}$ is the Poisson random measure with intensity $a C_{\beta} r^{-\beta-1} \mathrm{~d} \mathbf{x} \mathrm{d} r G(\mathrm{~d} m)$, and

$$
\widetilde{I}_{a}(\mu)=\int_{\mathbb{R}^{d} \times \mathbb{R} \times \mathbb{R}_{+}} a^{-1 / \alpha} m \mu\left[\tau_{\mathbf{x}, r} h\right] \widetilde{N}_{\beta, a}(\mathrm{~d} \mathbf{x}, \mathrm{d} r, \mathrm{~d} m) .
$$

We obtain:

$$
\sup _{\substack{\left\|\mu_{1}-\mu_{2}\right\|_{T V} \leq \delta \\ \mu_{1}, \mu_{2} \in \mathcal{A}}}\left|I_{a}\left(\mu_{1}-\mu_{2}\right)\right| \leq \widehat{I}_{a}\left(\mathfrak{m}_{\delta}\right):=\int|m| a^{-1 / \alpha} \mathfrak{m}_{\delta}\left[\tau_{\mathbf{x}, r}|h|\right] N_{\beta, a}(\mathrm{~d} \mathbf{x}, \mathrm{d} r, \mathrm{~d} m)
$$


and

$$
1-\left|\varphi_{\widehat{I}_{a}\left(\mathfrak{m}_{\delta}\right)}(\theta)\right|^{2} \leq 1-\exp \left(-2 C(G) C_{\beta}|\theta|^{\alpha} \int_{\mathbb{R}^{d} \times \mathbb{R}_{+}}\left|\mathfrak{m}_{\delta}\left[\tau_{\mathbf{x}, r}|h|\right]\right|^{\alpha} \mathrm{d} \mathbf{x} r^{-\beta-1} \mathrm{~d} r\right) .
$$

Using Lemma A.3 with $\gamma:=\alpha$ and plugging it with (3.12) into (3.3) with

$$
X=\sup _{\substack{\left\|\mu_{1}-\mu_{2}\right\|_{T V} \leq \delta \\ \mu_{1}, \mu_{2} \in \mathcal{A}}}\left|I_{a}\left(\mu_{1}-\mu_{2}\right)\right|
$$

we obtain

$$
\mathbb{E}\left[\left|\sup _{\substack{\left\|\mu_{1}-\mu_{2}\right\|_{T V} \leq \delta \\ \mu_{1}, \mu_{2} \in \mathcal{A}}}\right| \widetilde{I}_{a}\left(\mu_{1}\right)-\widetilde{I}_{a}\left(\mu_{2}\right)||^{\gamma}\right] \leq A(\gamma) A(\alpha, \gamma)\left(2 C(G) C_{\beta} C\left(\phi^{*}\right)\right)^{\gamma / \alpha} \delta^{\frac{(\alpha d-\beta) \gamma}{\alpha d}} .
$$

This allow to conclude the Proof of Proposition 2.2 like previously for Proposition 2.1.

\section{Discussion AND EXAMPLES}

\subsection{Fading function}

In this section, we shortly discuss the choice of the fading function $h$ satisfying $(H)$. Note that from a modeling point of view, it is natural to consider that the fading funtion $h$ is radial and non-increasing. In this case, $h$ is bounded and condition $\left(C_{\gamma}\right)$ for some $\gamma \in(1, \alpha)$ can be removed from our main result as explained in Remark 2.3. This is typically the case for the fading function $h=\mathbf{1}_{B(\mathbf{0}, 1)}$ which recovers the classical setting investigated in $[1,2,7]$. A general discussion of such fading effect is given in [6].

\subsection{Discrete configurations}

Our main results Propositions 2.1 and 2.2 are stated only for smooth configurations. Actually, when the configurations considered are discrete, typically $\mu=\delta_{\mathbf{y}}$, there is no hope to obtain functional convergence. Heuristically, the dependence between $M_{\rho}\left(\delta_{\mathbf{y}_{1}}\right)$ and $M_{\rho}\left(\delta_{\mathbf{y}_{2}}\right), \mathbf{y}_{1} \neq \mathbf{y}_{2}$, is given by the sum of the weights of the balls containing both points $\mathbf{y}_{1}$ and $\mathbf{y}_{2}$ using the so-called covariation (see [10], Sect. 2.7). But at the limit when $\rho \rightarrow 0$ (in the so-called zoom-out case), the rescaled balls turn to be very small and typically no ball contains simultaneously $\mathbf{y}_{\mathbf{1}}$ and $\mathbf{y}_{\mathbf{2}}$. This provides independence at the limit and we have:

$$
n_{0}(\rho)^{-1}\left(M_{\rho}\left(\delta_{\mathbf{y}}\right)-\mathbb{E}\left[M_{\rho}\left(\delta_{\mathbf{y}}\right)\right]\right) \stackrel{f d d}{\Longrightarrow} W_{\alpha}\left(\delta_{\mathbf{y}}\right), \quad \mathbf{y} \in \mathbb{R}^{d}
$$

where $W_{\alpha}\left(\delta_{\mathbf{y}}\right), \mathbf{y} \in \mathbb{R}^{d}$, are iid $S_{\alpha}\left(\sigma V^{1 / \alpha}, b, 0\right)$-distributed. Since the field $\left(W_{\alpha}\left(\delta_{\mathbf{y}}\right)\right)_{\mathbf{y} \in \mathbb{R}^{d}}$ consists of independent marginals, there is no hope for functional regularity. A similar remark can be fomulated when the configurations live in the space of measures $\mu$ with finite support. From a more technical point of view, Remark A.2 below indicates that our strategy, indeed, can not encompass discrete configurations.

\subsection{Compactness of the configurations}

In this section, we discuss the condition $(C 0)$. Since Propositions 2.1 and 2.2 are valid only for smooth configurations $\mu(\mathrm{d} \mathbf{y})=\phi_{\mu}(\mathbf{y})$ dy with $\phi_{\mu} \in L^{1}\left(\mathbb{R}^{d}\right) \cap L^{\alpha}\left(\mathbb{R}^{d}\right)$ the compactness of the set $\mathcal{A}$ of such configurations is characterized by the following result (see [4]):

Theorem 4.1 (Fréchet-Kolmogorov). Let $p \in\left[1,+\infty\left[\right.\right.$ and $\mathcal{S}$ be a subset of $L^{p}\left(\mathbb{R}^{d}\right)$. The set $\mathcal{S}$ is relatively compact if and only if:

(1) $\mathcal{S}$ is bounded; 
(2) $\lim _{r \rightarrow+\infty} \int_{\|\mathbf{x}\|>r}|f(\mathbf{x})|^{p} \mathrm{~d} \mathbf{x}=0$ uniformly on $\mathcal{S}$;

(3) and $\lim _{\mathbf{a} \rightarrow 0}\left\|\tau_{\mathbf{a}} f-f\right\|_{p}=0$ uniformly on $\mathcal{S}$, where $\tau_{\mathbf{a}} f(\mathbf{x})=f(\mathbf{x}-\mathbf{a})$.

A typical example is a family of uniform configurations on balls included in some fixed bounded ball $B \in \mathbb{R}^{d}$ :

$$
\mathcal{A}_{B}=\left\{\mu(\mathrm{d} \mathbf{x})=\mathbf{1}_{B(\mathbf{y}, r)}(\mathbf{x}) \mathrm{d} \mathbf{x}: B(\mathbf{y}, r) \subset B\right\} .
$$

The compactness in $L^{1}\left(\mathbb{R}^{d}\right) \cap L^{\alpha}\left(\mathbb{R}^{d}\right)$ comes from the Fréchet-Kolmogorov theorem since $\mathbf{1}_{B(\mathbf{y}, r)} \leq \mathbf{1}_{B}$ and $\mathbf{1}_{B} \in L^{1}\left(\mathbb{R}^{d}\right) \cap L^{\alpha}\left(\mathbb{R}^{d}\right)$ ensures (1) and (2) therein and (3) comes from that for all $B(\mathbf{y}, r) \subset B$, and $p=1, \alpha$,

$$
\begin{aligned}
\left\|\tau_{\mathbf{a}} \mathbf{1}_{B(\mathbf{y}, r)}-\mathbf{1}_{B(\mathbf{y}, r)}\right\|_{p}^{p} & =\int_{\mathbb{R}^{d}}\left|\mathbf{1}_{B(\mathbf{y}+a, r)}(\mathbf{x})-\mathbf{1}_{B(\mathbf{y}, r)}(\mathbf{x})\right|^{p} \mathrm{~d} \mathbf{x} \\
& =|B(\mathbf{y}+\mathbf{a}, r) \Delta B(\mathbf{y}, r)|=2|(B+\mathbf{a}) \backslash B| \longrightarrow 0, \mathbf{a} \rightarrow 0 .
\end{aligned}
$$

Another example with unbounded support configurations is

$$
\mathcal{A}_{\Lambda}=\left\{\mu(\mathrm{d} \mathbf{x})=\mathrm{e}^{-\lambda\|\mathbf{x}\|} \mathrm{d} \mathbf{x}: \lambda \in[\underline{\lambda}, \bar{\lambda}]\right\}
$$

with $0<\underline{\lambda}<\bar{\lambda}<+\infty$. The compactness in $L^{1}\left(\mathbb{R}^{d}\right) \cap L^{\alpha}\left(\mathbb{R}^{d}\right)$ comes from the Fréchet-Kolmogorov Theorem 4.1. Indeed, $\mathrm{e}^{-\lambda\|\mathbf{x}\|} \leq \mathrm{e}^{-\underline{\lambda}\|\mathbf{x}\|} \in L^{1}\left(\mathbb{R}^{d}\right) \cap L^{\alpha}\left(\mathbb{R}^{d}\right)$ ensures (1) and (2) in Theorem 4.1 and (3) therein comes for $p=1, \alpha$, from

$$
\begin{aligned}
& \left\|\tau_{\mathbf{a}} \mathrm{e}^{-\lambda\|\cdot\|}-\mathrm{e}^{-\lambda\|\cdot\|}\right\|_{p}^{p}=\int_{\mathbb{R}^{d}}\left|\mathrm{e}^{-\lambda \| \mathbf{x}-\mathbf{a} \mid}-\mathrm{e}^{-\lambda\|\mathbf{x}\|}\right|^{p} \mathrm{~d} \mathbf{x} \\
& \quad=\int_{\|\mathbf{x}\| \leq\|\mathbf{x}-\mathbf{a}\|}\left(\mathrm{e}^{-\lambda\|\mathbf{x}\|}-\mathrm{e}^{-\lambda\|\mathbf{x}-a\|}\right)^{p} \mathrm{~d} \mathbf{x}+\int_{\|\mathbf{x}-\mathbf{a}\| \leq\|\mathbf{x}\|}\left(\mathrm{e}^{-\lambda\|\mathbf{x}-\mathbf{a}\|}-\mathrm{e}^{-\lambda\|\mathbf{x}\|}\right)^{p} \mathrm{~d} \mathbf{x} \\
& \quad \leq \int_{\|x\| \leq\|\mathbf{x}-\mathbf{a}\|}\left(\mathrm{e}^{-\lambda\|\mathbf{x}\|}-\mathrm{e}^{-\lambda\|\mathbf{x}\|-\lambda\|\mathbf{a}\|}\right)^{p} \mathrm{~d} \mathbf{x}+\int_{\|\mathbf{x}-\mathbf{a}\| \leq\|\mathbf{x}\|}\left(\mathrm{e}^{-\lambda\|\mathbf{x}\|+\lambda\|a\|}-\mathrm{e}^{-\lambda\|x\|}\right)^{p} \mathrm{~d} \mathbf{x} \\
& \quad \leq\left(\mathrm{e}^{\bar{\lambda}\|\mathbf{a}\|}-1\right)^{p} \int_{\mathbb{R}^{d}} \mathrm{e}^{-\underline{\lambda} p\|\mathbf{x}\|} \mathrm{d} \mathbf{x}=\frac{(d-1) !}{(\underline{\lambda} p)^{d}}\left|B_{d}(\mathbf{0}, 1)\right|\left(\mathrm{e}^{\bar{\lambda}\|\mathbf{a}\|}-1\right)^{p} \longrightarrow 0, \quad \mathbf{a} \rightarrow 0,
\end{aligned}
$$

for all $\lambda \in[\underline{\lambda}, \bar{\lambda}]$ since $\left(1-\mathrm{e}^{-\lambda\|\mathbf{a}\|}\right) \leq\left(\mathrm{e}^{\lambda\|\mathbf{a}\|}-1\right) \leq\left(\mathrm{e}^{\bar{\lambda}\|\mathbf{a}\|}-1\right)$.

\subsection{Uniform control of the configurations}

In this section, we discuss the uniform control encapsulated into $\left(C_{p}\right), p=1, \gamma$ for some $\gamma \in(1, \alpha)$. Several sets of sufficients condition allow to satisfy $\left(C_{p}\right)$ easily.

In the standard case, we have $h=\mathbf{1}_{B(\mathbf{0}, 1)}$ (see [2,7]), and $\left(C_{p}\right)$ rewrites $|\mu|(B(\mathbf{x}, r)) \leq \mu^{*}(B(\mathbf{x}, r))$ for all ball $B(\mathbf{x}, r)$ and $\mu \in \mathcal{A}$. First, here are some examples of measures $\mu$ for which the conditions $\left(C_{1}\right)$ and $\left(C_{\gamma}\right)$ are met:

More generally, $\left(C_{p}\right)$ derives from $|\mu|(A) \leq \mu^{*}(A)$ for all Borelian set $A \subset \mathbb{R}^{d}$ by approximating $\left|h\left(r^{-1}(\mathbf{y}-\mathbf{x})\right)\right|^{p}$ by positive simple functions $\sum_{i} a_{i} \mathbf{1}_{A_{i}}(\mathbf{y})$ with $a_{i}>0$.

If the densities $\phi_{\mu}$ of the measures $\mu$ satisfy $\sup _{\mu \in \mathcal{A}}\left|\phi_{\mu}\right| \in L^{1}\left(\mathbb{R}^{d}\right) \cap L^{\alpha}\left(\mathbb{R}^{d}\right)$ then $\left(C_{p}\right)$ hold true. For instance, the conditions are satisfied if the measures $\mu$ are uniform measures with all their supports in a fixed bounded set. This covers Example 2.1 in [3].

The condition $\left(C_{p}\right)$ can also derive from a domination for a particular order associated to $h$ : for instance if $h$ is positive and convex, $\left(C_{p}\right)$ is obtained if all $\mu \in \mathcal{A}$ are dominated by $\mu^{*}$ for the convex-positive order (see [11]). 


\subsection{Typical examples}

Typical examples where all the conditions are met are the following:

- $h=\mathbf{1}_{B(\mathbf{0}, 1)}$ recovers the setting of $[1,2,7]$.

- $h$ is bounded and the set $\mathcal{A}$ of configurations is made of uniform measures on balls all included in a fixed bounded set, $c f$. (4.1).

- $h$ is bounded and the set $\mathcal{A}$ of configurations is given by (4.2).

\section{Appendix A.}

In this section, we prove technical bounds for $\mathfrak{m}_{\delta}\left[\tau_{\mathbf{x}, r}|h|\right]$ in terms of $\delta$ under different sets of condition. These bounds are the key point to derive the tightness of our processes. All along, we assume $(C 0)$ and $\left(C_{1}\right)$ and we assume also $\left(C_{\gamma}\right)$ for some $1<\gamma<\alpha$ unless $h$ is bounded in which case $\left(C_{\gamma}\right)$ is relaxed. Recall that $\mathfrak{m}_{\delta}\left[\tau_{\mathbf{x}, r}|h|\right]$ is defined in (3.7).

Lemma A.1. Let $\gamma \geq 1$.

(1) Assume the family $\mathcal{A}$ satisfies $\left(C_{\gamma}\right)$ and $h \in L^{\gamma}\left(\mathbb{R}^{d}\right)$. Then

$$
\int_{\mathbb{R}^{d}} \mathfrak{m}_{\delta}\left[\tau_{\mathbf{x}, r}|h|\right]^{\gamma} \mathrm{d} \mathbf{x} \leq 2 \delta^{\gamma-1} r^{d}\|h\|_{\gamma}^{\gamma} \mu^{*}\left(\mathbb{R}^{d}\right)
$$

(2) Assume the family $\mathcal{A}$ satisfies $\left(C_{1}\right)$ and $h \in L^{\infty}\left(\mathbb{R}^{d}\right) \cap L^{1}\left(\mathbb{R}^{d}\right)$. Then

$$
\int_{\mathbb{R}^{d}} \mathfrak{m}_{\delta}\left[\tau_{\mathbf{x}, r}|h|\right]^{\gamma} \mathrm{d} \mathbf{x} \leq 2 \delta^{\gamma-1} r^{d}\|h\|_{\infty}^{\gamma-1}\|h\|_{1} \mu^{*}\left(\mathbb{R}^{d}\right) .
$$

(3) Assume the family $\mathcal{A}$ satisfies $\left(C_{1}\right)$ and $h \in L^{1}\left(\mathbb{R}^{d}\right), \phi^{*} \in L^{\gamma}\left(\mathbb{R}^{d}\right)$. Then

$$
\int_{\mathbb{R}^{d}} \mathfrak{m}_{\delta}\left[\tau_{\mathbf{x}, r}|h|\right]^{\gamma} \mathrm{d} \mathbf{x} \leq r^{\gamma d}\left\|\phi^{*}\right\|_{\gamma}^{\gamma}\|h\|_{1}^{\gamma} .
$$

Proof. First, for (1): using below the Hölder's inequality, Condition $\left(C_{1}\right)$ and the Fubini's theorem in (A.5), we have:

$$
\begin{aligned}
\int_{\mathbb{R}^{d}} \mathfrak{m}_{\delta}\left[\tau_{\mathbf{x}, r}|h|\right]^{\gamma} \mathrm{d} \mathbf{x} & \leq \int_{\mathbb{R}^{d}\left\|\mu_{1}-\mu_{2}\right\|_{T V} \leq \delta} \sup _{\mu_{1}, \mu_{2} \in \mathcal{A}}\left(\left(\int_{\mathbb{R}^{d}}\left|h\left(r^{-1}(\mathbf{y}-\mathbf{x})\right)\right|^{\gamma}\left(\left|\mu_{1}-\mu_{2}\right|\right)(\mathrm{d} \mathbf{y})\right)\left(\left|\mu_{1}-\mu_{2}\right|\right)\left(\mathbb{R}^{d}\right)^{\gamma-1}\right) \mathrm{d} \mathbf{x} \\
& \leq 2 \delta^{\gamma-1} \int_{\mathbb{R}^{d}} \sup _{\mu \in \mathcal{A}}\left(\int_{\mathbb{R}^{d}}\left|h\left(r^{-1}(\mathbf{y}-\mathbf{x})\right)\right|^{\gamma}|\mu|(\mathrm{d} \mathbf{y})\right) \mathrm{d} \mathbf{x} \\
& =2 \delta^{\gamma-1} \int_{\mathbb{R}^{d}} \int_{\mathbb{R}^{d}}\left|h\left(r^{-1}(\mathbf{y}-\mathbf{x})\right)\right|^{\gamma} \mathrm{d} \mathbf{x} \mu^{*}(\mathrm{~d} \mathbf{y})=2 \delta^{\gamma-1} r^{d}\|h\|_{\gamma}^{\gamma} \mu^{*}\left(\mathbb{R}^{d}\right)
\end{aligned}
$$

which is (1) in the lemma. The proof of (2) follows the same lines using

$$
\left|h\left(r^{-1}(\mathbf{y}-\mathbf{x})\right)\right|^{\gamma} \leq\|h\|_{\infty}^{\gamma-1}\left|h\left(r^{-1}(\mathbf{y}-\mathbf{x})\right)\right|
$$

to replace, using $\left(C_{1}\right),(\mathrm{A} .4)$ by

$$
\int_{\mathbb{R}^{d}} \mathfrak{m}_{\delta}\left[\tau_{\mathbf{x}, r}|h|\right]^{\gamma} \mathrm{d} \mathbf{x} \leq 2 \delta^{\gamma-1}\|h\|_{\infty}^{\gamma-1} \int_{\mathbb{R}^{d}} \int_{\mathbb{R}^{d}}\left|h\left(r^{-1}(\mathbf{y}-\mathbf{x})\right)\right| \mathrm{d} \mathbf{x} \mu^{*}(\mathrm{~d} \mathbf{y}) .
$$


For (3), still using below Hölder's inequality but with $\phi \in L^{\gamma}\left(\mathbb{R}^{d}\right)$ and $h \in L^{1}\left(\mathbb{R}^{d}\right)$, and using $\left(C_{1}\right)$ and a change of variable in (A.6), we have:

$$
\begin{aligned}
\int_{\mathbb{R}^{d}} \mathfrak{m}_{\delta}\left[\tau_{\mathbf{x}, r}|h|\right]^{\gamma} \mathrm{d} \mathbf{x} & =\int_{\mathbb{R}^{d}} \sup _{\left\|\mu_{1}-\mu_{2}\right\|_{T V} \leq \delta}\left(\int_{\mathbb{R}^{d}} \mid h\left(\mu_{2} \in \mathcal{A}\right.\right. \\
& \leq r^{\gamma d} \int_{\mathbb{R}^{d}}\left|\int_{\mathbb{R}^{d}} h(\mathbf{y}) \phi^{*}(r \mathbf{y}+\mathbf{x}) \mathrm{d} \mathbf{y}\right|^{\gamma} \mathrm{d} \mathbf{x} \\
& \leq r^{\gamma d} \int_{\mathbb{R}^{d}}\left\{\left(\int_{\mathbb{R}^{d}}|h(\mathbf{y})| \mathrm{d} \mathbf{y}\right)^{\gamma-1} \int_{\mathbb{R}^{d}}\left|\phi^{*}(r \mathbf{y}+\mathbf{x})\right|^{\gamma}|h(\mathbf{y})| \mathrm{d} \mathbf{y}\right\} \mathrm{d} \mathbf{x} \\
& =r^{\gamma d}\left\|\phi^{*}\right\|_{\gamma}^{\gamma}\|h\|_{1}^{\gamma} \mathrm{d} \mathbf{x}
\end{aligned}
$$

with the Fubini theorem to derive the last equality.

Remark A.2. The measure $\mu^{*}$ is assumed to be smooth. A carefull reading of the previous proof indicates that this is used only to derive the bound $O\left(r^{\gamma d}\right)$ in (A.3). However, this bound fails if $\mu^{*}$ is no longer smooth: indeed, in the proof above, the bound $O\left(r^{\gamma d}\right)$ in (A.7) derives from (A.6) only if $\mu^{*}$ does not have atom: since if $\mu^{*}\left(\mathbf{y}_{0}\right)>0$, then (A.6) is bounded below by

$$
\int_{\mathbb{R}^{d}}\left|h\left(r^{-1}\left(\mathbf{y}_{0}-\mathbf{x}\right)\right)\right|^{\gamma} \mu^{*}\left(\mathbf{y}_{0}\right)^{\gamma} \mathrm{d} \mathbf{x}=r^{d} \mu^{*}\left(\mathbf{y}_{0}\right)^{\gamma} \int_{\mathbb{R}^{d}}|h(\mathbf{x})|^{\gamma} \mathrm{d} \mathbf{x}
$$

and there is no hope to obtain a bound in $r^{\gamma d}$ for small $r$.

Lemma A.3. Let $\gamma>\beta / d$ and assume $\mu^{*}(\mathrm{~d} \mathbf{y})=\phi^{*}(\mathbf{y}) \mathrm{d} \mathbf{y}$ with $\phi^{*} \in L^{1}\left(\mathbb{R}^{d}\right) \cap L^{\gamma}\left(\mathbb{R}^{d}\right)$. Assume moreover either $\left(C_{1}\right),\left(C_{\gamma}\right)$ and $h \in L^{1}\left(\mathbb{R}^{d}\right) \cap L^{\gamma}\left(\mathbb{R}^{d}\right)$ or only $\left(C_{1}\right)$ when $h \in L^{1}\left(\mathbb{R}^{d}\right) \cap L^{\infty}\left(\mathbb{R}^{d}\right)$. Then, for any $\rho>0$,

$$
\int_{\mathbb{R}^{d} \times \mathbb{R}_{+}} \mathfrak{m}_{\delta}\left[\tau_{\mathbf{x}, r}|h|\right]^{\gamma} \mathrm{d} \mathbf{x} F_{\rho}(\mathrm{d} r) \leq \rho^{\beta} \delta^{\frac{\gamma d-\beta}{d}} \frac{(\gamma-1) d}{(\beta-d)(\gamma d-\beta)} 2^{\frac{\gamma d-\beta}{(\gamma-1) d}}\left(\left\|\phi^{*}\right\|_{\gamma}\|h\|_{1}\right)^{\frac{\gamma(\beta-d)}{(\gamma-1) d}}\left(C^{\prime}\left(\phi^{*}\right)\right)^{\frac{(\gamma d-\beta) \gamma}{(\gamma-1) d}},
$$

where the constant $C^{\prime}\left(\phi^{*}\right)$ depends on the hypothesis in force, i.e.

$$
C^{\prime}\left(\phi^{*}\right)= \begin{cases}\left\|\phi^{*}\right\|_{1}^{1 / \gamma}\|h\|_{\gamma} & \text { when }\left(C_{1}\right) \text { and }\left(C_{\gamma}\right) \text { are satisfied, } \\ \left\|\phi^{*}\right\|_{1}^{1 / \gamma}\|h\|_{1}^{1-1 / \gamma}\|h\|_{\infty}^{1 / \gamma} \text { when }\left(C_{1}\right) \text { is satisfied and } h \in L^{\infty}\left(\mathbb{R}^{d}\right) .\end{cases}
$$

Proof. Assume $\left(C_{1}\right)$ and $\left(C_{\gamma}\right)$ are satisfied. Then, using (A.1) and (A.3) in Lemma A.1, we have using a change of variable:

$$
\begin{aligned}
\int_{\mathbb{R}_{+}} \int_{\mathbb{R}^{d}} \mathfrak{m}_{\delta}\left[\tau_{\mathbf{x}, r}|h|\right]^{\gamma} \mathrm{d} \mathbf{x} F_{\rho}(\mathrm{d} r) & \leq \int_{\mathbb{R}_{+}}\left(2 \delta^{\gamma-1} \rho^{\mathrm{d}} r^{d}\|h\|_{\gamma}^{\gamma}\left\|\phi^{*}\right\|_{1}\right) \wedge\left(\rho^{\gamma d} r^{\gamma d}\left\|\phi^{*}\right\|_{\gamma}^{\gamma}\|h\|_{1}^{\gamma}\right) F(\mathrm{~d} r) \\
& =\rho^{\gamma d}\left\|\phi^{*}\right\|_{\gamma}^{\gamma}\|h\|_{1}^{\gamma} \int_{0}^{\delta^{1 / d} c / \rho} r^{\gamma d} F(\mathrm{~d} r)+2 \delta^{\gamma-1} \rho^{d}\|h\|_{\gamma}^{\gamma}\left\|\phi^{*}\right\|_{1} \int_{\delta^{1 / d} c / \rho}^{+\infty} r^{d} F(\mathrm{~d} r)
\end{aligned}
$$

with

$$
c=\left(\frac{2\|h\|_{\gamma}^{\gamma}\left\|\phi^{*}\right\|_{1}}{\left\|\phi^{*}\right\|_{\gamma}^{\gamma}\|h\|_{1}^{\gamma}}\right)^{1 /((\gamma-1) d)} .
$$

Using now the bound on the truncated moments of $F$ given below in Lemma A.5 with $\delta:=\gamma d>\beta$ in (A.13) and $\delta:=d$ in (A.14), we obtain

$$
\int_{\mathbb{R}^{d}} \mathfrak{m}_{\delta}\left[\tau_{\mathbf{x}, r}|h|\right]^{\gamma} \mathrm{d} \mathbf{x} F_{\rho}(\mathrm{d} r) \leq \rho^{\beta}\left[\left\|\phi^{*}\right\|_{\gamma}^{\gamma}\|h\|_{1}^{\gamma} \frac{\beta}{\gamma d-\beta} C_{\beta} c^{\gamma d-\beta}+2 \delta^{\gamma-1}\|h\|_{\gamma}^{\gamma}\left\|\phi^{*}\right\|_{1} \frac{\beta}{\beta-d} C_{\beta} c^{d-\beta}\right]
$$

from which (A.8) comes after cancellation when $c$ is reported from (A.10). 
When only $\left(C_{1}\right)$ is satisfied and $h \in L^{1}\left(\mathbb{R}^{d}\right) \cap L^{\infty}\left(\mathbb{R}^{d}\right)$, the proof is similar using (A.2) from Lemma A.1 in place of (A.1).

Lemma A.4. Let $\gamma<\beta<\gamma d$ and assume $\mu^{*}(\mathrm{~d} \mathbf{y})=\phi^{*}(\mathbf{y}) \mathrm{d} \mathbf{y}$ with $\phi^{*} \in L^{1}\left(\mathbb{R}^{d}\right) \cap L^{\gamma}\left(\mathbb{R}^{d}\right)$. Assume moreover either $\left(C_{1}\right),\left(C_{\gamma}\right)$ and $h \in L^{1}\left(\mathbb{R}^{d}\right) \cap L^{\gamma}\left(\mathbb{R}^{d}\right)$ or only $\left(C_{1}\right)$ when $h \in L^{1}\left(\mathbb{R}^{d}\right) \cap L^{\infty}\left(\mathbb{R}^{d}\right)$. Then,

$$
\int_{\mathbb{R}^{d} \times \mathbb{R}_{+}}\left|\mathfrak{m}_{\delta}\left[\tau_{\mathbf{x}, r}|h|\right]\right|^{\gamma} \mathrm{d} \mathbf{x} r^{-\beta-1} \mathrm{~d} r \leq \delta^{\frac{\gamma d-\beta}{d}} \frac{(\gamma-1) d}{(\beta-d)(\gamma d-\beta)} 2^{\frac{\gamma d-\beta}{(\gamma-1) d}}\left(\left\|\phi^{*}\right\|_{\gamma}\|h\|_{1}\right)^{\frac{\gamma(\beta-d)}{(\gamma-1) d}}\left(C^{\prime}\left(\phi^{*}\right)\right)^{\frac{(\gamma d-\beta) \gamma}{(\gamma-1) d}},
$$

where the constant $C^{\prime}\left(\phi^{*}\right)$ is given like in Lemma A.3 in (A.9).

Proof. Assume $\left(C_{1}\right)$ and $\left(C_{\gamma}\right)$ are in force. Then, using (A.1) and (A.3) in Lemma A.1, we have

$$
\begin{aligned}
\int_{\mathbb{R}^{d} \times \mathbb{R}_{+}}\left|\mathfrak{m}_{\delta}\left[\tau_{\mathbf{x}, r}|h|\right]\right|^{\gamma} \mathrm{d} \mathbf{x} r^{-\beta-1} \mathrm{~d} r & \leq \int_{\mathbb{R}^{d}}\left(2 \delta^{\gamma-1}\|h\|_{\gamma}^{\gamma}\left\|\phi^{*}\right\|_{1} r^{d-\beta-1}\right) \wedge\left(\left\|\phi^{*}\right\|_{\gamma}^{\gamma}\|h\|_{1}^{\gamma} r^{\gamma d-\beta-1}\right) \mathrm{d} r \\
& =\left\|\phi^{*}\right\|_{\gamma}^{\gamma}\|h\|_{1}^{\gamma} \int_{0}^{\delta^{1 / d} c} r^{\gamma d-\beta-1} \mathrm{~d} r+2 \delta^{\gamma-1}\|h\|_{\gamma}^{\gamma}\left\|\phi^{*}\right\|_{1} \int_{\delta^{1 / d} c}^{+\infty} r^{d-\beta-1} \mathrm{~d} r
\end{aligned}
$$

where $c$ is given in (A.10). Computing the integral, we obtain (A.11) after cancellation. When $\left(C_{1}\right)$ is in force with $h$ bounded, similar computations yields (A.11) with now (A.2) and (A.3) in Lemma A.1.

The following bounds are easily computed (see [3] for details):

Lemma A.5. For $\delta>0$, when $u \rightarrow+\infty$, we have:

$$
\int_{0}^{u} r^{\delta} F(\mathrm{~d} r) \sim \begin{cases}C s t & \text { if } \delta<\beta \\ \beta C_{\beta} \ln u & \text { if } \delta=\beta \\ \frac{\beta}{\delta-\beta} C_{\beta} u^{\delta-\beta} & \text { if } \delta>\beta\end{cases}
$$

and for $0<\delta<\beta$, when $u \rightarrow+\infty$, we have:

$$
\int_{u}^{+\infty} r^{\delta} F(\mathrm{~d} r) \sim \frac{\beta}{\beta-\delta} C_{\beta} u^{\delta-\beta}
$$

Moreover when $\delta>\beta$, we have the global bound

$$
\int_{0}^{u} r^{\delta} F(\mathrm{~d} r) \leq C u^{\delta-\beta}
$$

Acknowledgements. Both authors thank an anonymous referee for valuable comments on a preleminary version of the manuscript.

\section{REFERENCES}

[1] H. Biermé, A. Estrade and I. Kaj, Self-similar random fields and rescaled random balls models. J. Theoret. Probab. 23 (2010) 1110-1141.

[2] J.-C. Breton and C. Dombry, Rescaled weighted random balls models and stable self-similar random fields. Stochastic Process. Appl. 119 (2009) 3633-3652.

[3] J.-C. Breton and C. Dombry, Functional macroscopic behavior of weighted random ball model. Alea, Lat. Am. J. Probab. Math. Stat. 8 (2011) 177-196.

[4] H. Brézis, Analyse fonctionnelle. Masson (1983).

[5] W. Feller, An Introduction to Probability Theory and its Applications. Wiley (1966), Vol. 2. 
[6] I. Kaj, Aspects of wireless network modeling based on Poisson point processes Fields. Institute Workshop on Applied Probability, Carleton University. Available at: http://www.math.uu.se/ ikaj/preprints/wirelessnotes2.pdf (2006).

[7] I. Kaj, L. Leskelä, I. Norros and V. Schmidt, Scaling limits for random fields with long-range dependence. Ann. Probab. 35 (2007) 528-550.

[8] O. Kallenberg, Foundations of Modern Probability, 2nd edition. Springer, New-York (2002).

[9] T. Mikosch, S. Resnick, H. Rootzén and A. Stegeman, Is network traffic approximated by stable Lévy motion of fractional Brownian motion. Ann. Appl. Probab. 12 (2002) 23-68.

[10] G. Samorodnitsky and M.S. Taqqu, Stable non-Gaussian random processes. Stochastic Modeling, Chapman \& Hall (1994).

[11] M. Shaked and G. Shanthikumar, Stochastic orders and their applications. Probability and Mathematical Statistics. Academic Press (1994).

[12] B. von Bahr and C.-G. Esseen, Inequalities for the $r$ th absolute moment of a sum of random variables, $1 \leq r \leq 2$. Ann. Math. Statist. 36 (1965) 299-303.

[13] X. Yang and A.P. Petropulu, Co-Channel interference modeling in a Poisson field of interferers in wireless communications. IEEE Trans. Signal Process. 51 (2003) 64-76. 